\title{
The creep behavior of simple structures with a stress range dependent constitutive model
}

\author{
James T Boyle \\ Department of Mechanical Engineering, University of Strathclyde, Glasgow, \\ Scotland, G1 $1 X J$ \\ Phone: +441415482311 Fax:+441415525105 E-mail jim.boyle@strath.ac.uk
}

\begin{abstract}
High temperature design remains an issue for many components in a variety of industries. Although finite element analysis for creep is now an accessible tool, most analyses outside the research domain use long standing and very simple constitutive models - in particular based on a power law representation. However for many years it has been known that a range of materials exhibit different behaviors at low and moderate stress levels.. Recently studies of the behavior of high temperature structures with such a stress range dependent constitutive model have begun to emerge. The aim of this paper is to examine further the detailed behavior of simple structures with a modified power law constitutive model in order to instigate a deeper understanding of such a constitutive model's effect on stress and deformation and the implications for high temperature design. The structures examined are elementary - a beam in bending and a pressurized thick cylinder - but have long been used to demonstrate the basic characteristics of nonlinear creep.
\end{abstract}

Keywords Creep - Stress range dependent constitutive model - Structural analysis - High temperature design

\section{Introduction}

Stress analysis for creep has a long history in engineering mechanics driven by the needs of design for high temperature in many industries but primarily power generation and aerospace and a growing need to ensure the reliability of solder joints in electronics packaging. In the absence of the computing power required for detailed finite element analysis of time-dependent nonlinear creep with complex loading histories, robust simplified methods of analysis were developed $[1,2,3]$ for simple constitutive models. These simple constitutive models, for example the time- and strain-hardening constitutive equations, were based on adaptations for time-varying stress of equally simple models for the secondary creep stage from constant load/stress uniaxial tests where minimum creep rate is 
constant. The most common secondary creep constitutive model has been the Norton-Bailey Law which gives a power-law relationship between minimum creep rate and (constant) stress. The unique mathematical properties of the power law allowed the development of robust simplified methods, many of which can be found in high temperature design codes. Now that detailed finite element analysis for creep is readily accomplished on the desktop it is perhaps surprising that the simple time- or strain-hardening constitutive models based on power law creep remain the most widely available in common commercial finite element software, such as ANSYS or ABAQUS, even though more comprehensive time-dependent nonlinear constitutive models are available (and can be included as user-defined materials). The most common reason for persisting with the more simple constitutive models is the ease with which material constants can be derived from experiments, the ability to check detailed solutions with simplified (robust) methods and an underlying understanding of the expected behavior of simple (but fundamental) structures subject to power law creep $[1,2,3]$. Nevertheless it has long been known that creep over a range of stress does not follow one simple power law relationship, typically (approximately) following one power law at low stress and another at high stress - a phenomenon known as 'power-law breakdown'. A common observation is a shift from a power law (usually dislocation) mechanism at 'moderate' stress to a diffusion mechanism at 'low' stress, characterized by a linear viscous relationship between creep rate and stress [4,5] with a more significant power-law breakdown at 'high' stress. Such a stress range dependent constitutive model, with a transition from linear to power law behavior, has recently been studied by Naumenko, Altenbach and Gorash $[6,7,8]$ : stress analyses using this modified power law were compared to linear and pure power law over a range of stress and load for several simple structures.

In this paper the analyses of Naumenko, Altenbach \& Gorash described in [6] are extended to provide a more detailed representation of the behavior of two simple structures - the beam in bending and a pressurized thick cylinder - using this modified power law, in particular to add a study of the deformation characteristics and the role of the simplified methods of analysis developed previously for pure power law creep. 


\section{Secondary creep constitutive model}

The minimum creep rate ( $\dot{\varepsilon}_{\text {min }}$ ) during the secondary (or steady state) deformation stage is often related to the (constant) applied stress $(\sigma)$ by a power law relationship in the form

$$
\dot{\varepsilon}_{\min }=B \sigma^{n}
$$

where $B$ and $n$ are constants determined from uniaxial creep testing. Use of a power law relation reflects an almost linear relationship between $\log ($ minimum creep rate) and $\log$ (stress) which is often found in creep tests: typical results for an austenitic stainless steel AISI 316L(N) taken from Rieth et al [9] are shown in Fig. 1.

However many metals and alloys typically exhibit different regimes with $n \approx 1$ at low stresses and $n \approx 4$ or 5 at higher stress levels with $n$ increasing again in the power law breakdown regime [4]. This is illustrated in Fig. 2, taken from [4] based on data on 0.5Cr0.5Mo0.25V steel from Evans et al. [10]. Indeed at lower temperatures (although still above that for creep) even the data from [9] shows similar behavior, Fig.3. Numerous attempts have been made to find a continuous curve to describe this behavior over the complete stress range, principal amongst these being the hyperbolic sine relationship

$$
\dot{\varepsilon}_{\text {min }}=B \sinh (C \sigma)
$$

and the equation proposed by Garofalo [11]

$$
\dot{\varepsilon}_{\text {min }}=B \sinh (C \sigma)^{n}
$$

where $B, C$ and $n$ are constants. A more complete summary can be found in [12]. It is often argued that the change in behavior from low to moderate stress can be explained by diffusional creep theories, while the transition from moderate to high stress (power law breakdown) can be accounted for by diffusion-controlled mechanisms (such as diffusion through or along grain boundaries and movement of lattice dislocations, for example in pure metals. These explanations are not generally agreed [4]. Nevertheless there remains an obvious need to perform stress analysis with this type of constitutive behavior and reliable (if not perfect) constitutive models are required. Williams \& Wilshire proposed the 'transition stress' model [13] for power law breakdown 


$$
\dot{\varepsilon}_{\text {min }}=B\left(\sigma-\sigma_{0}\right)^{p}
$$

where $B$ and $p$ are constants and $\sigma_{p}$ is the transition stress. Unfortunately the transition stress cannot be measured reliably. For the transition from low to moderate stress Naumenko et al [6] proposed a constitutive relationship which assumed that the physical mechanisms were independent and that the corresponding creep rates could simply be added:

$$
\frac{\dot{\varepsilon}_{\min }}{\dot{\varepsilon}_{0}}=\frac{\sigma}{\sigma_{0}}+\left(\frac{\sigma}{\sigma_{0}}\right)^{n}
$$

where $\sigma_{0}, \dot{\varepsilon}_{0}$ and $n$ are material constants. The stress $\sigma_{0}$ is a different kind of transition stress from that of Williams \& Wilshire since it specifies the stress level at which the behavior changes from linear (viscous) to power law, Fig. 4. Eqn. 5, which we shall refer to as a 'modified power law' for simplicity, was used by Naumenko, Altenbach and Gorash [6,7] to examine how the stress system in simple components - uniaxial stress relaxation, a beam in bending and a pressurized thick cylinder - would change as compared to purely linear and purely power-law behavior. In this paper the beam and thick cylinder problems will be re-examined in more detail - the stress relaxation problem will be considered elsewhere in the context of a more detailed analysis of elastic follow-up [14].

\section{Simple component behavior}

\subsection{Pure bending of a beam}

The classic problem of the pure bending of a rectangular cross section $(b \times h)$ beam with a modified power law (stress range dependent constitutive model) subjected to a bending moment $M$ has been considered by Naumenko, Altenbach \& Gorash [6]. The geometry and loading are as shown in Fig.5. In the following, the notation from [6] is essentially maintained, but with minor variations.

Under a constant applied bending moment under secondary creep the rate of curvature $\dot{\chi}$ of the center-line of the beam is related, assuming pure bending, to the axial (longitudinal) creep strain rate

$$
\dot{\varepsilon}_{x}=\dot{\chi} z
$$


where $z$ is measured from the center-line as shown in Fig. 5.

The bending moment is related to the axial stress $\sigma_{x}$ through the equilibrium equation

$$
M=2 b \int_{0}^{h / 2} \sigma_{x} z d z
$$

The constitutive equation is taken as Eqn.(5).

As in [6], dimensionless (normalized) variables are defined as

$$
\xi=\frac{2 z}{h} \quad s=\frac{\sigma}{\sigma_{0}} \quad \dot{e}=\frac{\dot{\varepsilon}_{x}}{\dot{\varepsilon}_{0}}=\dot{\kappa} \xi \quad \dot{\kappa}=\frac{\dot{\chi} h}{2 \dot{\varepsilon}_{0}}
$$

Introducing a dimensionless load factor $\lambda=\frac{M}{W \sigma_{0}}$, where $W=\frac{b h^{2}}{6}$ is the section moment, Eqns. (5), (6) \& (7) can be rewritten and combined as:

$$
\dot{e}=\dot{\kappa} \xi=f(s) \quad \lambda=3 \int_{0}^{1} s \xi d \xi
$$

where $f(s)=s+s^{n}$.

Then the rate of change of normalized curvature $\dot{\kappa}$ can be found, for a prescribed load factor $\lambda$ and creep exponent $n$, from a solution of the non-linear equation

$$
3 \int_{0}^{1} f^{-1}(\dot{\kappa} \xi) \xi d \xi-\lambda=0
$$

which is expressed in a different, but equivalent, form in [6]. The longitudinal stress distribution $\mathrm{s}(\xi)$ as it varies with through the thickness $\xi$ of the beam is then derived from the solution of the non-linear equation

$$
s(\xi)=f^{-1}(\dot{\kappa} \xi)
$$

For pure power law creep, ignoring the linear viscous part in Eqn. (5), the solution to Eqns. (8) \& (9) corresponds (using the current normalization scheme) to the familiar steady state creep solution for a beam in bending [1, 2, 3, 12]:

$$
\dot{\kappa}=\left(\frac{2 n+1}{3 n} \lambda\right)^{n} \quad s=\frac{2 n+1}{3 n} \lambda \xi^{1 / n}
$$

For pure linear (viscous) behavior, the solution is equivalent to that for linear elasticity

$$
\dot{\kappa}=\lambda \quad s=\lambda \xi
$$


We can then interpret the load factor $\lambda$ as the ratio of the maximum linear (elastic) stress in the beam to the transition stress $\sigma_{0}$. Of course both the classic steady state and linear elastic solutions can be normalized such that the normalized curvature rate and longitudinal stress are independent of the load factor $\lambda$, but this is not done for the modified stress range dependent constitutive equation, Eqn.(5).

\subsection{Pressurized thick cylinder}

The classic problem of a pressurized thick cylinder was also considered by Naumenko, Altenbach \& Gorash [6,7] as an example of secondary creep using a modified power law under multi-axial stress. In the following the notation of [6] is repeated, again with minor variations:

Assume that the cylinder is long and uniformly heated, with an inner radius $a$ and an outer radius $b$, as shown in Fig. 6 and that plane strain conditions prevail. The geometry of the cylinder is best described by a cylindrical polar coordinate system $(r, \varphi, z)$; then let the principal strain rates be $\left(\dot{\varepsilon}_{r}, \dot{\varepsilon}_{\varphi}, \dot{\varepsilon}_{z}\right)$, the principal stresses be $\left(\sigma_{r}, \sigma_{\varphi}, \sigma_{z}\right)$ and $\bar{\sigma}(r)$ the von-Mises equivalent stress. The hoop and radial displacement rates are given by $\dot{v}_{\varphi}$ and $\dot{v}_{r}$ respectively. The boundary conditions at the inner and outer surfaces are given by

$$
\sigma_{r}(a)=-p \quad \sigma_{r}(b)=0
$$

where $p$ is the internal pressure.

The solution procedure for this problem is well established [1, 2, 3, 12] and relies on the condition of volume constancy (incompressibility)

$$
\dot{\varepsilon}_{\varphi}=\frac{\dot{v}_{r}}{r}, \quad \dot{\varepsilon}_{r}=\frac{d \dot{v}_{r}}{d r} \quad \dot{\varepsilon}_{\varphi}+\dot{\varepsilon}_{r}=0
$$

to reveal that the radial displacement rate has the simple form

$$
\dot{v}_{r}=\frac{C}{r}
$$

where $C$ is an integration constant. 
For the conditions of radially symmetric plane strain it may be shown that the hoop strain rate can be related to the equivalent von Mises stress using the modified power law, Eqn.(5) by

$$
\frac{\dot{\varepsilon}_{\varphi}}{\dot{\varepsilon}_{0}}=\frac{\sqrt{3}}{2}\left(\frac{\bar{\sigma}}{\sigma_{0}}+\left(\frac{\bar{\sigma}}{\sigma_{0}}\right)^{n}\right)
$$

which may be combined with the first of Eqns.(11) and the boundary conditions (10) to obtain a nonlinear equation for the constant $C$ (the details can be found in [6] or [7]).

By introducing the dimensionless (normalized) variables

$$
\eta=\frac{r}{a} \quad s=\frac{\bar{\sigma}}{\sigma_{0}} \quad c=\frac{C}{\dot{\varepsilon}_{0} a^{2} \sqrt{3}} \quad \rho=\frac{b}{a}
$$

together with a load factor $\lambda=\frac{p}{\sigma_{0}}$ we obtain, by combining Eqns.(10-13), the following nonlinear equation for $c$ :

$$
\int_{1}^{\rho} \frac{2}{\eta^{2}} f^{-1}\left(\frac{c}{\eta^{2}}\right) d \eta=\sqrt{3} \lambda
$$

where $f(s)=s+s^{n}$.

For a prescribed radius ratio $\rho$, power exponent $n$, and load factor $\lambda$ the normalized constant $c$ can be obtained. Then the hoop and radial stress distributions, $\sigma_{\varphi}(\eta), \sigma_{r}(\eta)$, as they vary with $\eta$, can be obtained as:

$$
\begin{aligned}
& \frac{\sigma_{\varphi}(\eta)}{p}=\frac{1}{\lambda} \frac{2}{\sqrt{3}}\left(f^{-1}\left(\frac{c}{\eta^{2}}\right)+\int_{\rho}^{\eta} \frac{1}{\eta} f^{-1}\left(\frac{c}{\eta^{2}}\right) d \eta\right) \\
& \frac{\sigma_{r}(\eta)}{p}=\frac{1}{\lambda} \frac{2}{\sqrt{3}}\left(\int_{\rho}^{\eta} \frac{1}{\eta} f^{-1}\left(\frac{c}{\eta^{2}}\right) d \eta\right)
\end{aligned}
$$

respectively. As with the beam in bending the results will depend upon the load factor $\lambda$.

The normalized maximum radial displacement rate occurs at the inside surface from Eqn.(12) and can be shown to be 


$$
\frac{\dot{v}_{r, \max }}{a \dot{\varepsilon}_{0}}=\sqrt{3} c
$$

Due to the simplicity of the creep strain rates, also from Eqn.(12) the maximum hoop and radial stress also occur at the inside and are proportional to $\dot{v}_{r \text {,max }}$.

Finally, the equivalent normalized stress distributions corresponding to pure power law can be obtained as [1, 2, 3, 12]:

$$
\frac{\sigma_{\varphi}(\eta)}{p}=\frac{\left(\left(\frac{1}{\rho}\right)^{\frac{2}{n}}-\left(\frac{n-2}{n}\right)\left(\frac{1}{\eta}\right)^{\frac{2}{n}}\right)}{\left(1-\left(\frac{1}{\rho}\right)^{\frac{2}{n}}\right)} \quad \frac{\sigma_{r}(\eta)}{p}=\frac{\left(\left(\frac{1}{\rho}\right)^{\frac{2}{n}}-\left(\frac{1}{\eta}\right)^{\frac{2}{n}}\right)}{\left(1-\left(\frac{1}{\rho}\right)^{\frac{2}{n}}\right)}
$$

The linear (viscous) solution can be obtained from the above with $n=1$.

\section{Results}

It is required to use some form of numerical analysis to solve each of the preceding problems: the beam in bending, Eqns. (8) \& (9), and the pressurized thick cylinder, Eqns.(14), (15) \& (16). Here both Mathcad and Matlab were used for the numerical analysis to ensure converged independently checked solutions. In their study of these two problems Naumenko, Altenbach \& Gorash [6] focused on the effect of the stress range constitutive model change in each component's behavior compared to the limiting cases of linear (viscous) behavior and pure power law creep. They usefully identified for each case an approximate limiting stress/load level above which the pure power law could be applied and showed that below this load level the stress distributions were affected by linear (viscous) behavior. A few representative stress distributions were presented for each case. In this paper a more detailed study of the behavior of these simple structures using a modified power law is presented including a more detailed discussion of the effect on stress distributions, deformation characteristics (which were not covered in [6]) and the role of simplified methods of analysis. 


\subsection{Pure bending of a beam}

The normalized curvature rate, $\dot{\kappa}$, and axial stress distribution, $s(\xi)$, are found from a solution of Eqns.(8) \& (9). Solutions depend upon the load factor, $\lambda$, and power exponent, $n$, in this case.

As in [6] the normalized stress distribution $\frac{\sigma(\xi)}{M / W}=\frac{s}{\lambda}$ can be plotted from the beam centerline to the outer fiber ( $\xi=1$ ); this normalized stress corresponds to the ratio between the computed stress and the maximum linear (equivalent elastic) stress. Results are shown in Fig.7 for $n=3,7 \& 12$ for various values of the load factor $\lambda$ together with the pure linear and pure power law stresses. It can be seen that the stress distributions tend to the pure linear solution as the value of $\lambda$ decreases and the pure power solution as the value of $\lambda$ increases. For higher values of the power index $n$ the stress distribution tends to be almost identical to the pure power law solution as the value of $\lambda$ increases; however for smaller values of $n$ the maximum stress at the outer fiber remains larger than the pure power law. This can also be seen in a plot of the variation of maximum normalized stress at the outer fiber with load factor $\lambda$, Fig.8. Even at high values of the load factor, where a large part of the stress distribution would be well above the transition stress and in the power law regime, for lower values of the power exponent $n$ the linear viscous component of the constitutive equation still has an effect - a feature which is not apparent from [6]. Further, Fig.7 clearly shows the presence of a 'skeletal point' [1]. The skeletal point is a point through the thickness where the stress is virtually independent of the creep law used. It was first noted by Anderson et al. [15] The presence of a skeletal point in structures subject to creep was important to the development of 'reference stress' methodology for high temperature (creep) design [16,17]. The skeletal point concept was later extended by Seshadri [18] who demonstrated the existence of multiple such points - called R-nodes - in complex components which could be used not only for the estimation of behavior at high temperature but also for simplified estimation of limit loads. The significance of the results shown in Fig.7 are that the location of the skeletal point, and the corresponding normalized stress value, are almost independent of the load factor $\lambda$ as well. Of course the (unnormalized) stress at the skeletal point does depend on the load factor, being 
proportional to the applied load. The skeletal point could be seen in the results reported in [6] but its significance was not noted.

Further, in pure power law creep it has been observed that the maximum stress in a component typically exhibits a linear variation with the reciprocal of the power exponent, $n,[1,19]$. For the beam in bending, the maximum normalized stress at the outer fiber, is exactly obtained as

$$
\frac{\sigma_{\max }}{M / W}=\frac{2}{3}+\frac{1}{n}
$$

in which the linear variation with $1 / n$ is evident. The limiting values of $n=1$ and $n \rightarrow \infty$ can be shown to correspond to linear elastic and perfectly-plastic maximum stress. This feature of the pure power law has been demonstrated for many components and load conditions [1] (a recent example is the peak stress in an oval pressurized pipe bend [20]). Fig.9 plots the variation of maximum normalized stress with $1 / n$ for various values of the load factor $\lambda$ for the modified power law. It can be seen that provided the load factor $\lambda>1$ the normalized maximum stress decreases from $n=1$ as $1 / n$ decreases towards $n \rightarrow \infty$. The variation tends towards linear with $1 / n$ as the load factor increases. The following may also be observed if $\lambda>1$ : (a) in all cases as $n \rightarrow 1$ the normalized maximum stress tends to 1 , (b) in all cases as $n \rightarrow \infty$ the maximum stress (seems to) tend to a value of 2/3, as the pure power law. As a consequence it may be concluded that, to a good (engineering) approximation, above a certain value of load factor the maximum stress variation with power exponent using a modified power law can be well approximated by the pure power law solution, which itself can be well estimated from linear elastic and perfectly plastic solutions.

The study reported in [6] did not consider the deformation characteristics of the modified power law. Here the behavior of the normalized curvature rate will be examined in more detail. Plots of normalized curvature rate, $\dot{\kappa}$, against the load factor, $\lambda$, for various values of the power exponent $n$ are shown in Fig.10 - part (a) shows the complete set of plots for the range of variables studied, while part (b) focuses on a smaller region using a log/log plot. Fig.11 shows the equivalent pure power law solution 


$$
\dot{\kappa}=\left(\frac{2 n+1}{3 n} \lambda\right)^{n}
$$

for comparison. It can be seen that for values of the load factor less than a 'crossover' point just less than $\lambda=1.5$ the variation of curvature rate with load factor is almost linear and the linear viscous part of the constitutive equation dominates. This behavior can be further seen in Fig. 12 where curvature rate is plotted against the power index $n$ for various values of the load factor - part (a) shows the complete set of plots for the range of variables studied, while part (b) focuses on a smaller region with a $\log / \log$ plot. It can be seen that for $\lambda<1.5$ the curvature rate is almost independent of the power index $n$. This feature is perhaps curious since the stress distributions for such values of load factor are different from the equivalent linear distributions and the normalized longitudinal strain rate is proportional to the curvature rate, $\dot{e}=\dot{\kappa} \xi$. This observation prompted an examination of the relation between the curvature rates (and therefore also strain rates) from the pure linear, pure power law and modified power law constitutive relations. It turns out that, depending on the load factor, deformations using the modified power law can be reasonably estimated by simply adding the deformations from pure linear and pure power law in this example. If the load factor is close to (and less than) the 'cross-over' value for the load factor from Fig. 10 then this simple estimation can underestimate by up to $20 \%$ for large values of the power exponent $n$, but is much less otherwise as shown in Fig.13. This is an attractive observation: variations in strain rate predictions due to creep testing, scatter and curve fitting can have similar error levels and the implication (at least in this simple example) is that deformation rates for a material modeled by the modified power law could simply be obtained from superimposing equivalent linear deformations and pure power law creep deformations. The latter could be estimated for design purposes using reference stress or other robust simplified methods,. (High temperature creep design rules are more typically based on deformation rather than stress in the first instance). 


\subsection{Pressurized thick cylinder}

The simple thick cylinder problem allows a study of creep under a multi-axial stress state. For a modified power law, the solution of Eqns. (14), (15) \& (16) depends not only on the power index $n$ and load factor $\lambda$ as for the beam in bending, but also on a geometry factor - the radius ratio $\rho$. The cylinder geometry can therefore be varied from moderately-thin to thick: in this study $\rho$ varies takes values of 1.3, 2.0 and 3.0. In the following study only the circumferential (hoop) stress will be considered; the radial stress, due to the nature of the boundary conditions, Eqns. (10), varies in a simple fashion from $-p$ to zero. As a basis for comparison, Figs. 14 (a) shows an example of the hoop stress distribution, $\sigma_{\varphi} / p$, corresponding to a pure power law, for various values of the power index $n$, and to a linear viscous law $(n=1)$ from Eqns.(17) for $\rho=2.0$. This plot can be used toform the basis of comparisons of stress distribution changes due to the effect of using the modified power law, where the load factor $\lambda$ must be taken into consideration. As is well known, the hoop stress variation through the thickness of the cylinder in all three cases changes from the pure linear case, where the maximum stress is at the inside surface, to the pure steady state, where the maximum stress is at the outside, as can be seen from Fig. 14(a) These limiting cases then may be compared to the hoop stress distributions as the load factor $\lambda$ varies from $0.2,0.7,0.9 \& 2.0$; these are not reproduced in full here but can be found in Online Resource 1. Examining each of the plots of Online Resource 1 it can be seen that as the load factor increases the hoop stress distribution changes from a form similar to pure linear to that of the pure power law. For the moderately thick cylinder ( $\rho=1.3$ ) the change occurs between load factors $0.2 \&$ 0.7, Fig. 14 (b) \& (c), while for the two thick cylinders ( $\rho=2.0 \& 3.0)$ the change occurs between load factors 0.7 \& 0.9, Fig 14(d) \& (e), and 0.9 \& 2.0 respectively. More detailed studies show that for the case $\rho=3.0$ the change occurs between load factor $0.9 \& 1.3$. It is clear then (this was perhaps not immediately clear from [6]) that the influence of the linear component of the modified power law is more evident as the thickness increases in the sense that the power law component does not dominate until higher values of the load factor. This reflects the fact that the overall stress magnitude reduces as the thickness increases. Further, as in the case of the beam in bending, an approximate 'skeletal 
point' can be observed: the position of this point moves outwards as the cylinder thickness increases while the magnitude of the stress at this point decreases. Close to the values of load factor at which the behavior changes from being more dominated by the power law component than the linear component of the modified power law the skeletal point is perhaps less distinct, but arguably the trend is clear enough for engineering design purposes, as discussed for the beam in bending. In fact if the von-Mises equivalent stress $\bar{\sigma} / p$ is plotted instead, the skeletal point becomes more distinct. Finally, as in the case of the beam in bending, the position of the skeletal point and magnitude of the corresponding normalized stress value are again almost independent of the load factor $\lambda$ with the (un-normalized) stress being proportional to the applied pressure at the skeletal point.

The study of the beam in bending in Sec. 4.1 showed that the maximum stress exhibited an almost linear variation with the reciprocal of the power index, $1 / n$. This observation would need more careful interpretation for the thick cylinder since, as discussed from Fig. 14, the position of the maximum hoop stress changes from the inner to the outer surface when considering pure linear and pure power law. Fig. 15 plots the (normalized) outer surface stress as a function of $1 / n$ (with $n$ in the range 3 to 20) for various values of the load factor and for $\rho=1.3,2.0 \&$ 3.0. It can be seen that in each case, above some transition value of the load factor, the outer stress is indeed sensibly linear with $1 / n$. The limiting values of the classical pure linear $n \rightarrow 1$ and pure power law $n \rightarrow \infty$ are also indicated on Fig.15 and it is apparent that the calculated results for the modified power law, again above some transition value of the load factor, do tend to these limiting values.

Finally, as for the beam in bending, the study reported in [6] did not consider the deformation characteristics for the thick cylinder. The normalized maximum displacement at the inside surface, Eqn.(16), is plotted in Fig.16 against the load factor $\lambda$ for various values of $n$ - a log-log scale is used for discussion purposes. Three values of thickness are used as before and in each case, similar to the beam in bending, there is a cross-over value of load factor below which the variation of maximum displacement is almost linear with respect to the load factor (this can be 
better verified using a linear scale on each axis). Above the cross-over point the variation of the (log) maximum displacement is effectively linear with respect to the (log) load factor, suggesting a power relationship and the possible existence of a reference stress. The cross-over point itself corresponds to that noted for the hoop stress distribution as it changes from a form similar to pure linear to that of the pure power law. For the moderately thick cylinder ( $\rho=1.3$ ) the change occurs between load factors $0.2 \& 0.7$, for the smaller thick cylinders $(\rho=2.0)$ the change occurs between load factors $0.7 \& 0.9$ and for the larger thick cylinder ( $\rho$ $=3.0$ ) the change occurs between load factor $0.9 \& 1.3$. Further, the maximum displacement can, with care, be reasonably estimated from a simple summation of the pure power law solution:

$$
\frac{\dot{v}_{r, \max }}{a \dot{\varepsilon}_{0}}=\sqrt{3}\left[\frac{\sqrt{3}}{n}\left(\frac{1}{1-\rho^{-2 / n}}\right) \lambda\right]^{n}
$$

with the linear solution, $n=1$ in the above. The percentage error is shown in Fig. 17. As was the case with the beam in bending if the load factor is close to (and less than) the 'cross-over' value for the load factor from the maximum displacement plot, Fig.16, then this simple estimation can underestimate by up to $20 \%$ for large values of the power exponent $n$, but is much less otherwise. The worst case corresponds to the thickest cylinder, $\rho=3.0$ with the error reducing with thickness. These observations are also valid for the maximum (normalized) strain rates from Eqns.(11). As a result, the conclusions reached for the deformation characteristics of the beam in bending with the modified power law are equally applicable in this simple structure with a multi-axial stress state.

\section{Discussion \& Conclusions}

This paper contains a more detailed study of work by Naumenko, Altenbach and Gorash [6,7] on how the stress systems in simple components - a beam in bending and a pressurized thick cylinder - are affected by using a modified power law, Eqn.(5), which contains both linear viscous and power law components. Naumenko et al showed that the stress systems in both components had limiting 
cases for low and high stress, namely the pure viscous and pure power law respectively. Between these limiting cases the (normalized) stress distributions depended not only on the exponent in the power law, but also some load factor since the modified power law solutions cannot be normalized to eliminate applied load. In this study the analysis has been extended to examine the role of simplified methods for estimating stress for design purposes and also to investigate the effect of the modified power law on deformation rates.

It is clear that the stress distributions for the beam in bending, Fig.7, and thick cylinder, Fig.14, change considerably from the limiting cases of pure linear viscous and pure power law, especially as the respective load factor changes, especially close to the transition from linear dominance to power law dominance. Nevertheless some characteristic features of the stress distributions found for pure power law can still be seen in both components: (i) the presence of a skeletal point $[15,16]$, where the normalized stress value is almost independent of the exponent in the power law and also the load factor, and (ii) above some transition load factor the maximum stress is approximately linear with respect to the reciprocal of the power exponent, $1 / n$, with the limiting cases of $n \rightarrow 1$ and $n \rightarrow \infty$ remaining the linear viscous solution and perfectly plastic solutions respectively, as in the case of the pure power law [19]. The latter seems to be valid even though large parts of the component continue to be dominated by the linear component, although the maximum stress location is dominated by the power law component. It has also been demonstrated that the maximum displacement and strain rates in these two components can be sensibly estimated through a superposition of the pure linear and pure power law solutions, except for load factors close to the transition from linear to power law dominance coupled with high values of the power exponent, $n$, Figs $13 \& 17$. The worst estimates are no more than $20 \%$ lower than the true solution and for a fairly wide range of load factor no more than $10 \%$ - this being well within currently acceptable bounds for scatter in creep data. There is the clear implication that maximum deformation rates can be reasonably estimated (as a lower bound in both examples) from superposition of linear and power law solutions, the latter allowing simplified estimates using reference stress, linear elastic and perfectly plastic limit solutions [1-3]. 
In conclusion, it is apparent that the simple robust methods developed for the estimation of maximum stress and deformation rate of components subject to pure power law creep [1-3] continue to have some relevance for the modified power law. Further work in the area of steady creep therefore includes a verification of these results for more complex structures using detailed finite element analysis and a more detailed study of the range of applicability of the simplified methods. Indeed, this study has only considered steady creep under constant load and gives no consideration of relaxation and redistribution of stress. Naumenko et al [6] briefly examined relaxation of a bar using the modified creep law, demonstrating that a stress range dependent creep law could have significant effect. That example has been extended by the author [14] to more complex structures undergoing creep relaxation, in particular elastic follow-up: it is shown there that a stress range dependent creep law wholly alters the time dependent structural response, unlike the steady creep cases described herein where familiar features are retained.

\section{References}

1. Boyle, J.T., Spence, J.: Stress Analysis for Creep. Butterworths, London (1983)

2. Penny, R.K., Marriott, D.L.: Design for Creep. Chapman \& Hall, London (1995)

3. Kraus, H.: Creep Analysis. Wiley, New York (1980)

4. Wilshire, B.: Observations, theories and predictions of high temperature creep behavior. Mettal. \& Mater. Trans. 33A:241-248 (2002)

5. Frost, H.J., Ashby, M.F.: Deformation-Mechanism Maps. Pergamon, Oxford (1982)

6. Naumenko, K., Altenbach, H., Gorash, Y.: Creep analysis with a stress range dependent constitutive model. Arch. Appl. Mech. 79:619-630 (2009)

7. Altenbach, H., Gorash, Y., Naumenko, K.: Steady-state creep of a pressurized thick cylinder in both the linear and power law ranges. Acta. Mech. 196:263-274 (2008)

8. Altenbach, H., Naumenko, K.,Gorash Y.: creep analysis for a wide stress range based on stress relaxation experiments. Int. J. Modern Physics 22B:5413-5418 (2008)

9. Reith, M. et al: Creep of the Austenitic Steel AISI 316 L(N). Forschungszentrum Karlsruhe in der Helmholtz-Gemeinschaft, Wissenschaftliche Berichte FZKA 7065 (2004)

10. Evans, R.W., Parker J.D., Wilshire B.: In: Wilshire B., Owen D.R.J. (eds.) Recent Advances in Creep and Fracture of Engineering Materials and Structures; 135-184 (1982)

11. Garofalo, F.: An empirical relation defining the stress dependence of minimum creep rate in metals. Trans. the Metall . Soc. AIME 227:351-356 (1963) 
12. Naumenko, K., Altenbach, H.: Modelling of Creep for Structural Analysis. Springer, Berlin (2007)

13. Williams, K. R., Wilshire, B.: On the Stress- and Temperature-Dependence of Creep of Nimonic 80A. Met. Sci. J. 7:176-179 (1973)

14. Boyle, J.T.: Stress relaxation and elastic follow-up using a stress range dependent constitutive model (Submitted)

15. Anderson, R.G. et al: Deformation of uniformly loaded beams obeying complex creep laws. Journ. Mech. Eng. Sci. 5:238-244 (1963)

16. Anderson, R. G.: Some observations on reference stresses, skeletal points, limit loads and finite elements. In: Creep in Structures, 3rd IUTAM Symposium, Leicester 166-178 (1981) 17. Boyle, J.T., Seshadri R.: The reference stress method in creep design: a thirty year retrospective. General Lecture, In: Proc IUTAM Symposium on Creep in Structures, Nagoya, Ed. S Murakami \& N Ohno, 297-311, Kluwer (2000)

18. Seshadri, R.: Inelastic evaluation of mechanical and structural components using the generalized local stress strain method of analysis. Nuclear Engineering and Design 153:287-303 (1995)

19. Calladine, C.R.: A rapid method for estimating the greatest stress in a structure subject to creep. Proc. IMechE. Conf. Thermal Loading and Creep (1964)

20. Yaghi, A.H.,Hyde, T.H., Becker, A.A., Sun, W.: Parametric peak stress functions of $90^{\circ}$ pipe bends with ovality under steady-state creep conditions. Int. Journ. Press. Vess. Piping 86:684-692 (2009) 


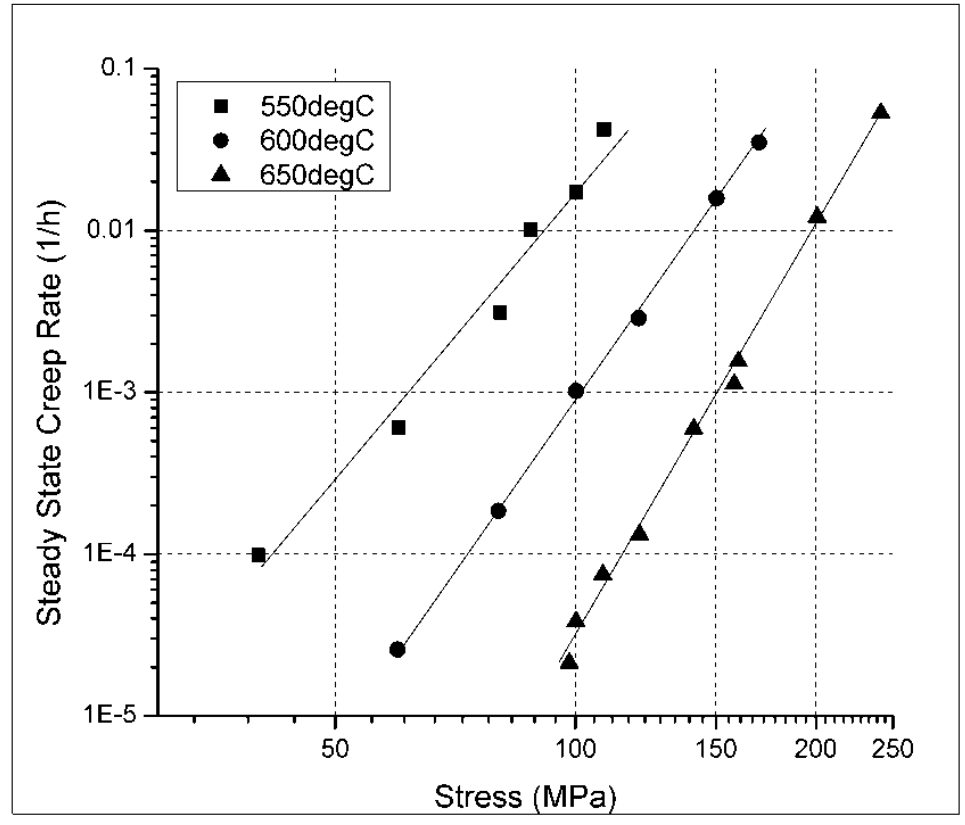

Fig. 1 Steady creep of austenitic AISI 316 L(N) 550-650 ${ }^{\circ} \mathrm{C}$ after [9]

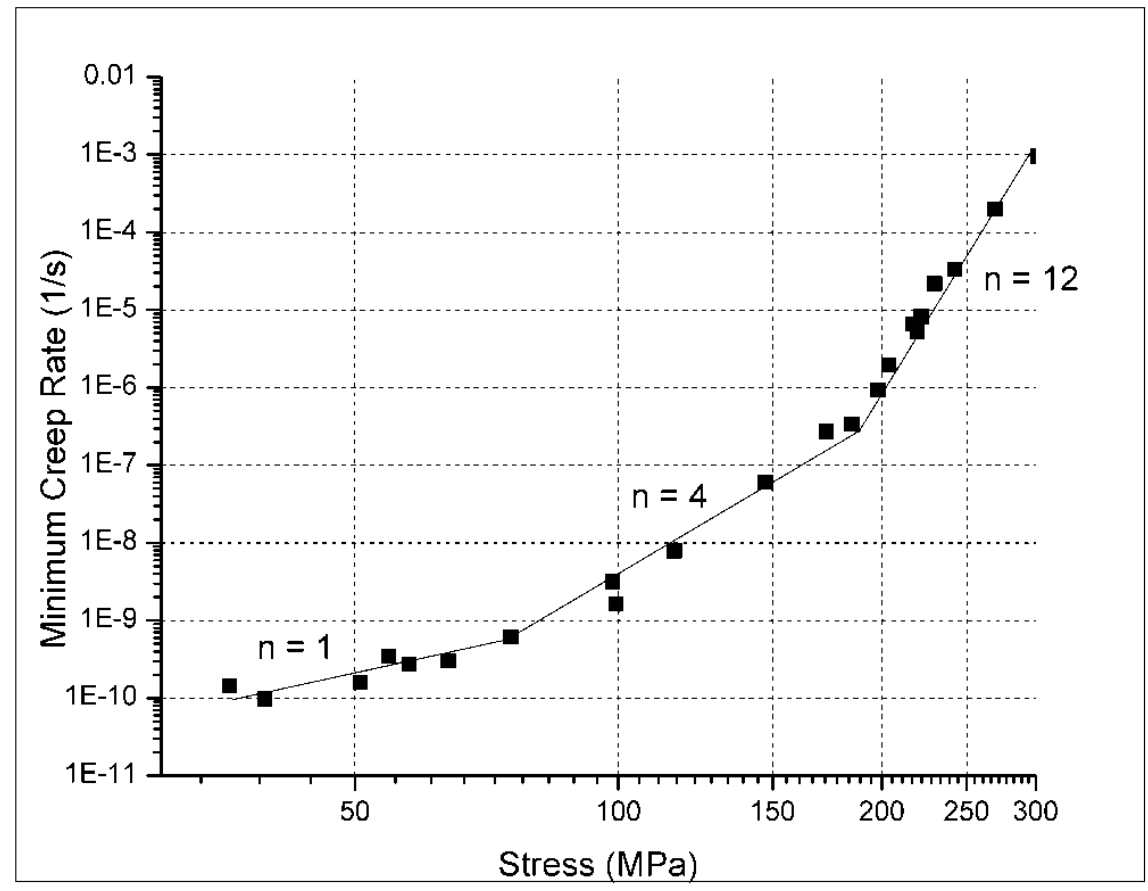

Fig. 2 Steady creep of $0.5 \mathrm{Cr} 0.5 \mathrm{Mo} 0.25 \mathrm{~V}$ after [4] 


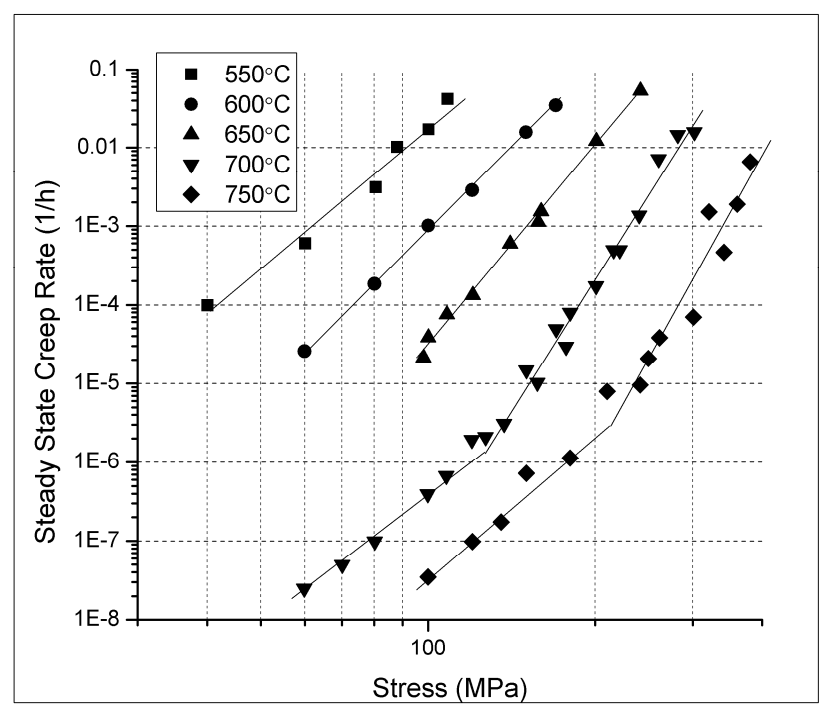

Fig. 3 Steady creep of austenitic AISI 316 L(N) 550-750 ${ }^{\circ} \mathrm{C}$ after [9]

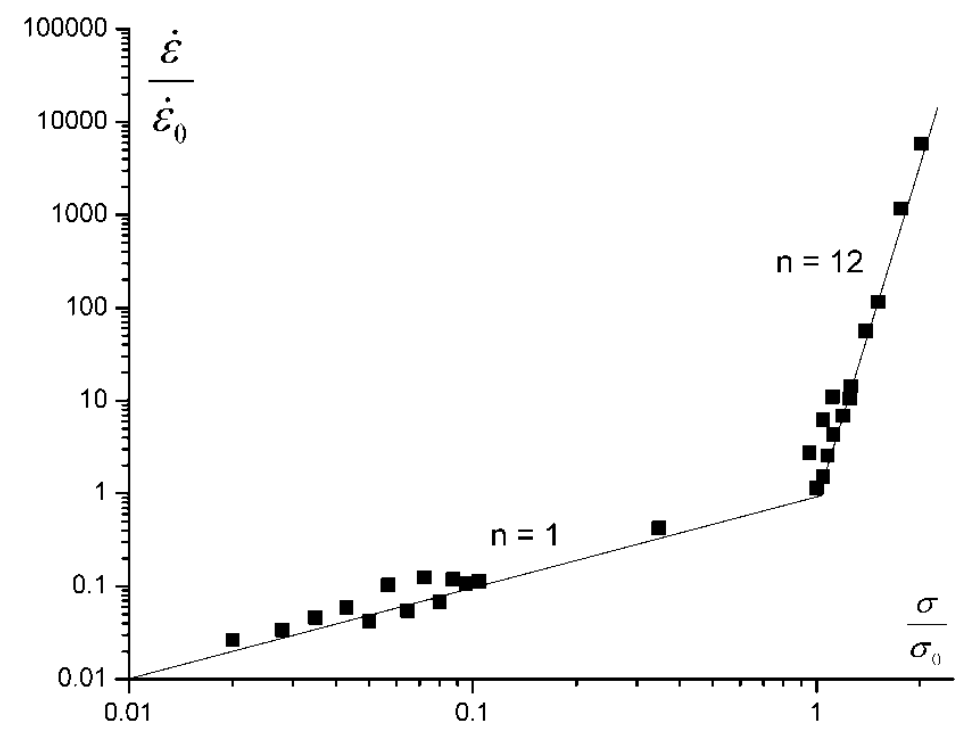

Fig. 4 Steady creep 9\%Cr steel at $600^{\circ} \mathrm{C}$ after [6] 


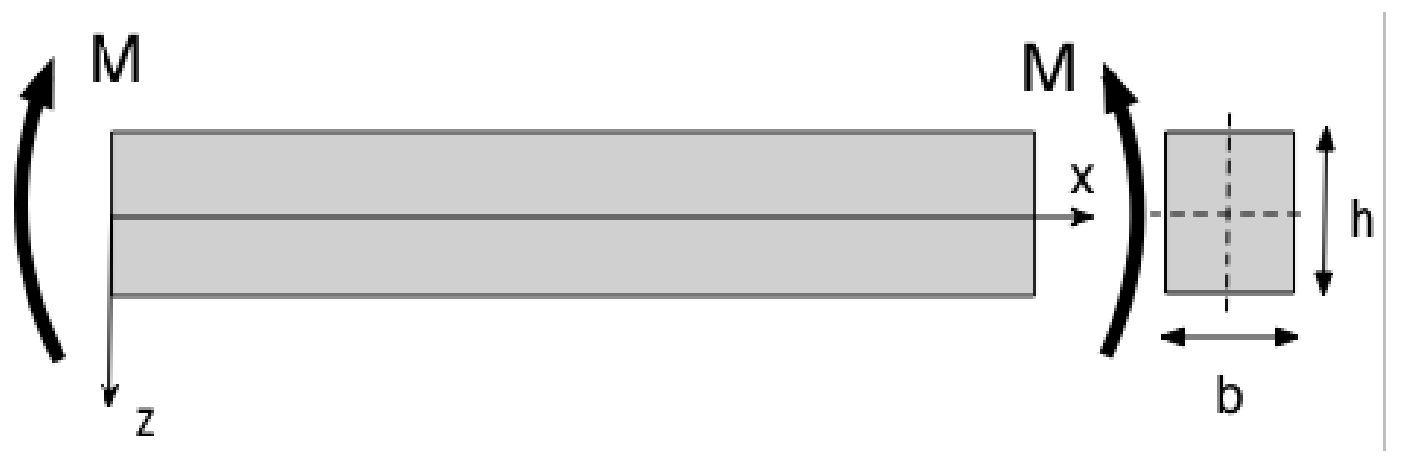

Fig. 5 Geometry and loading of a beam with rectangular cross section

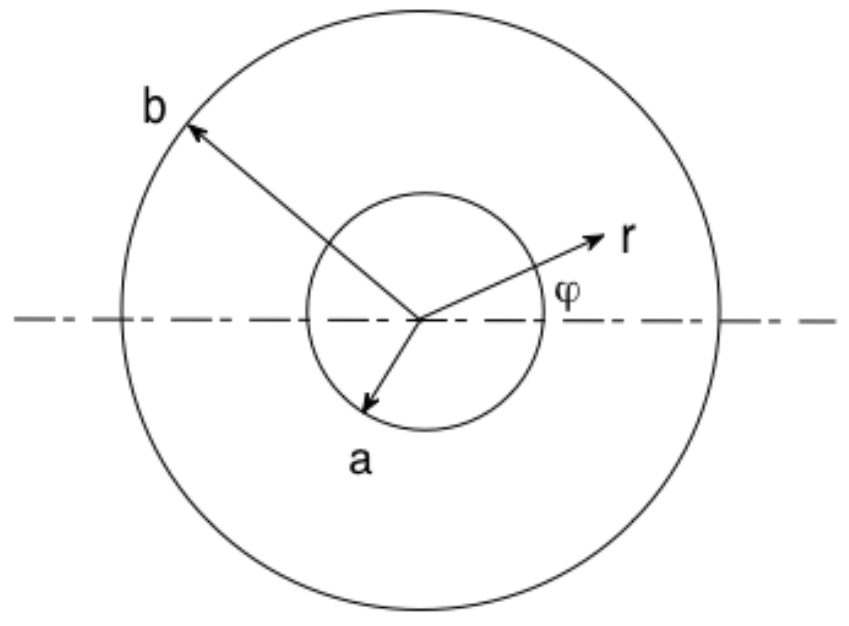

Fig. 6 Geometry and loading of a pressurized thick cylinder 

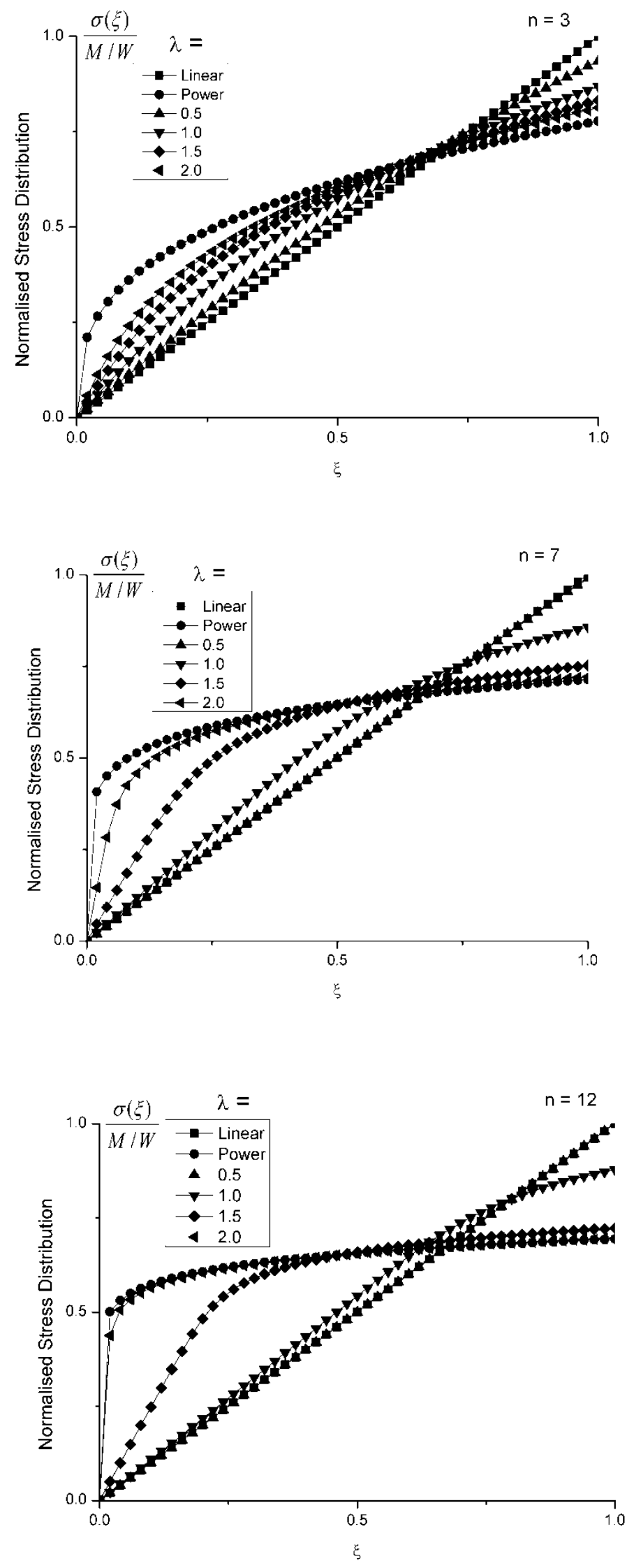

Fig. 7 Normalized stress distributions for beam in bending, $n=3,7 \& 12$ 


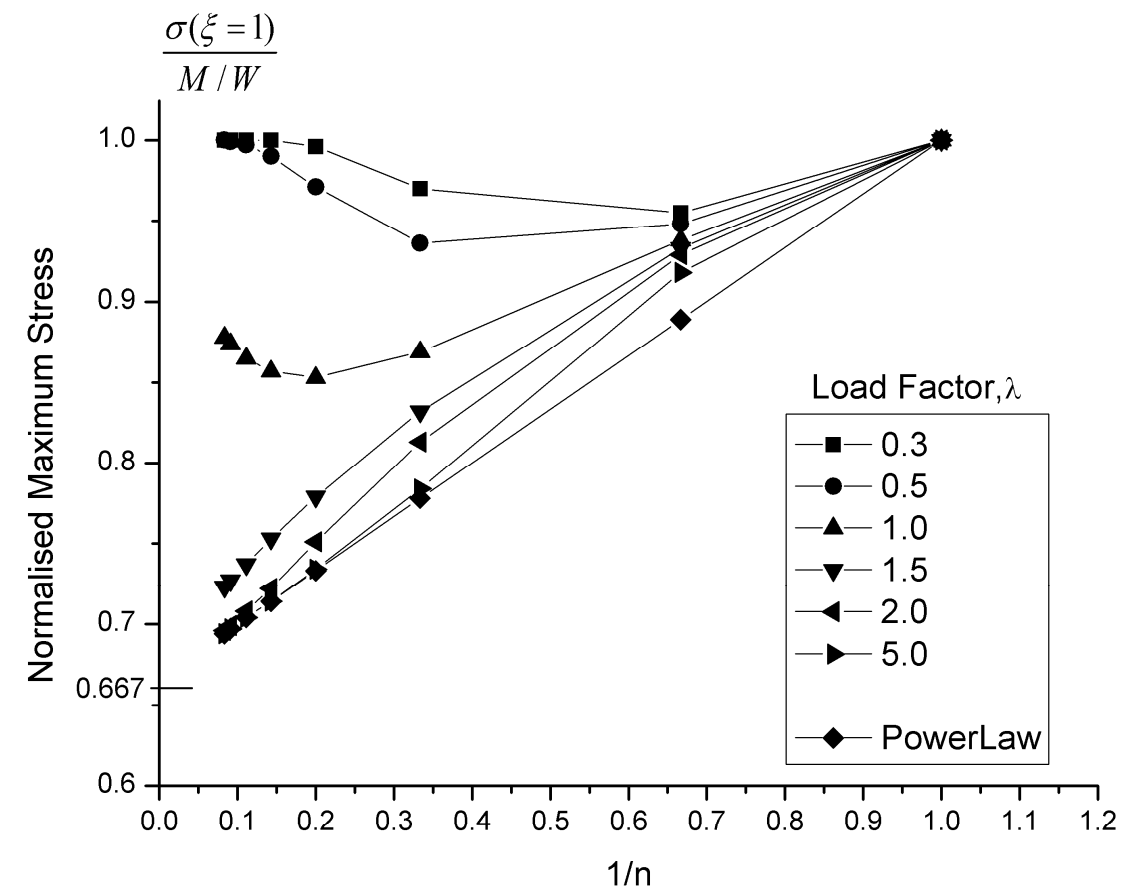

Fig. 8 Variation of maximum normalized stress with load factor

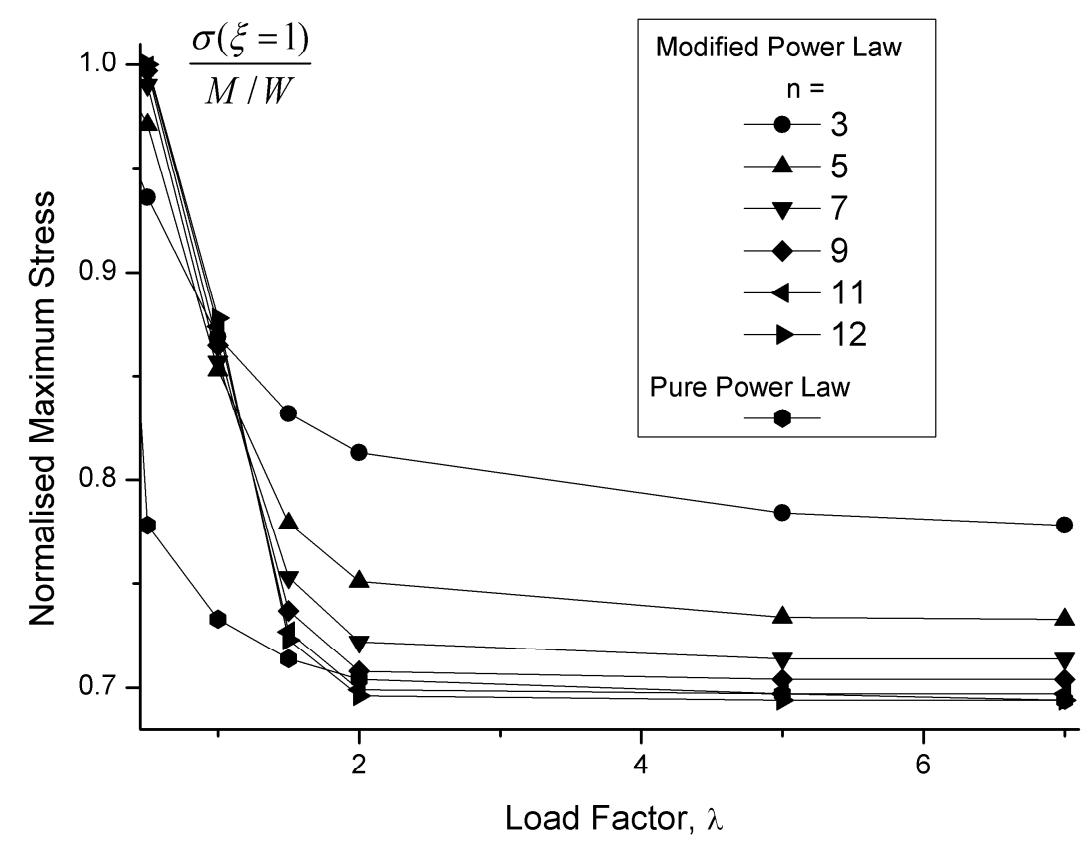

Fig. 9 Variation of maximum normalized stress with load $1 / n$ 


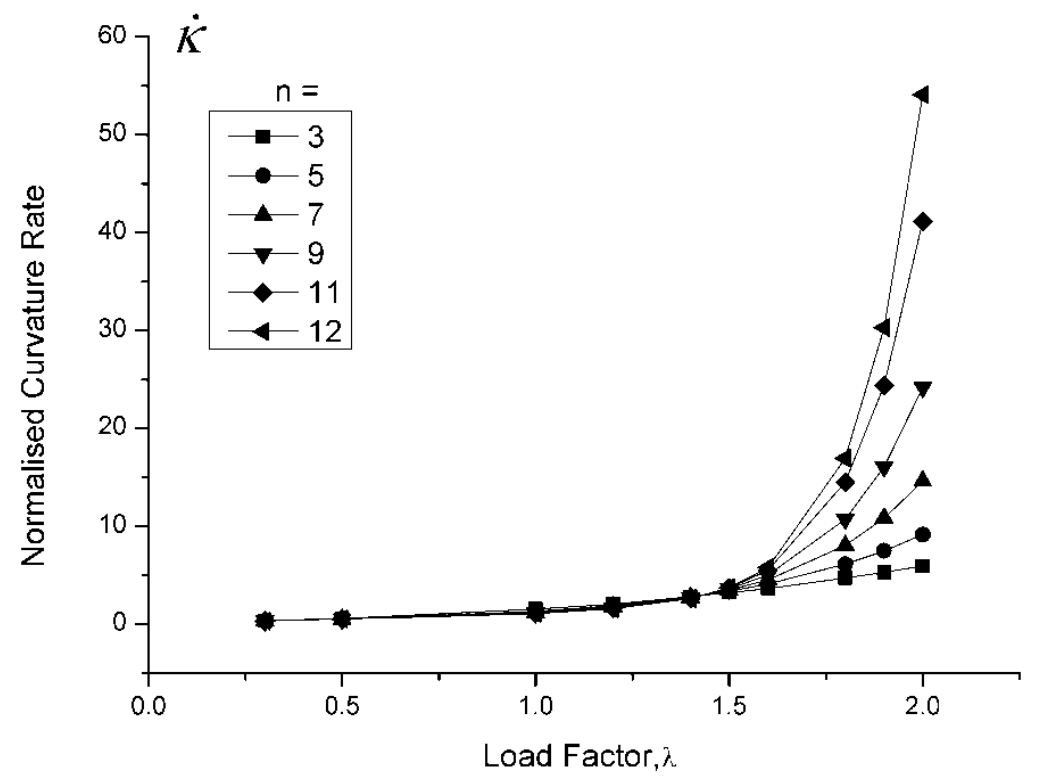

Fig. 10a Variation of maximum normalized curvature rate with load factor

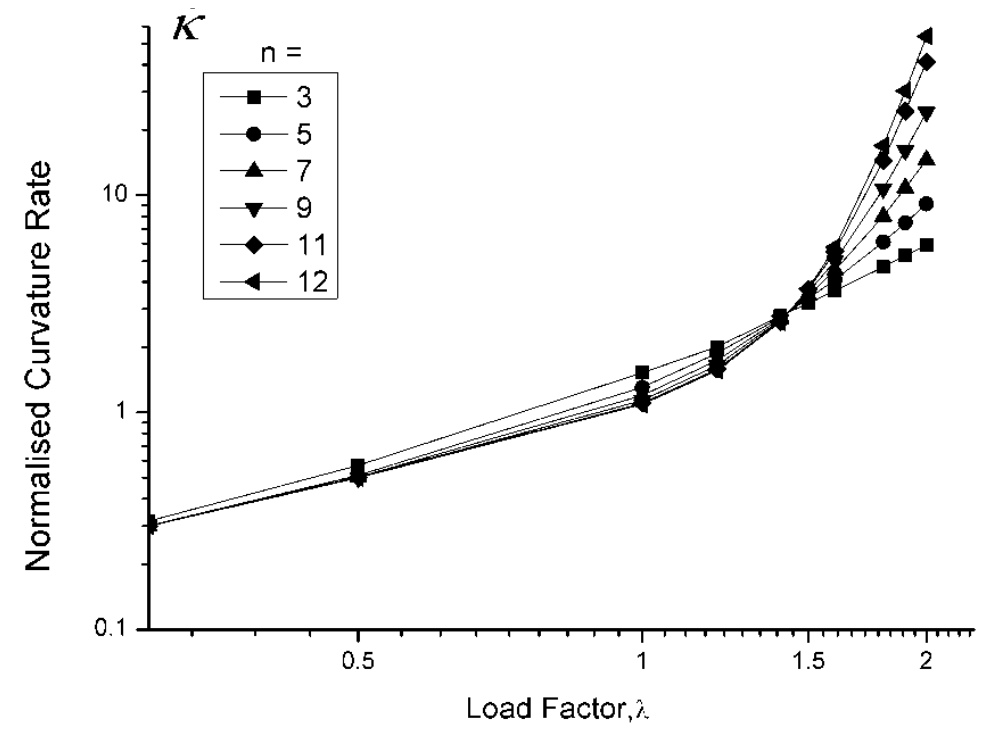

Fig. 10b Variation of maximum normalized curvature rate with load factor: zoomed view with $\log / \log$ plot

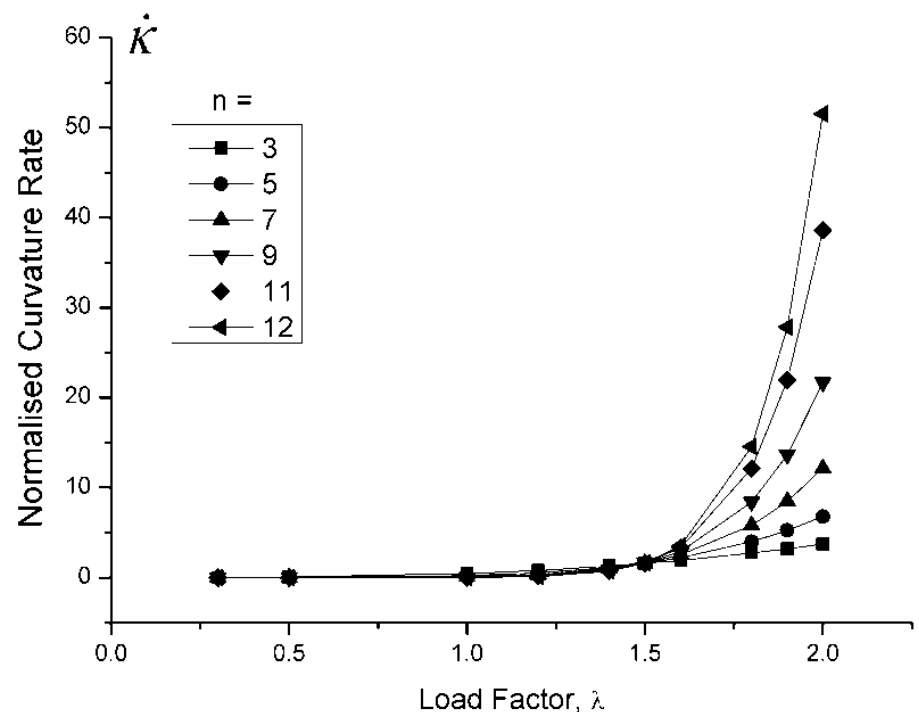

Fig. 11 Variation of maximum normalized curvature rate with load factor: pure power law creep 


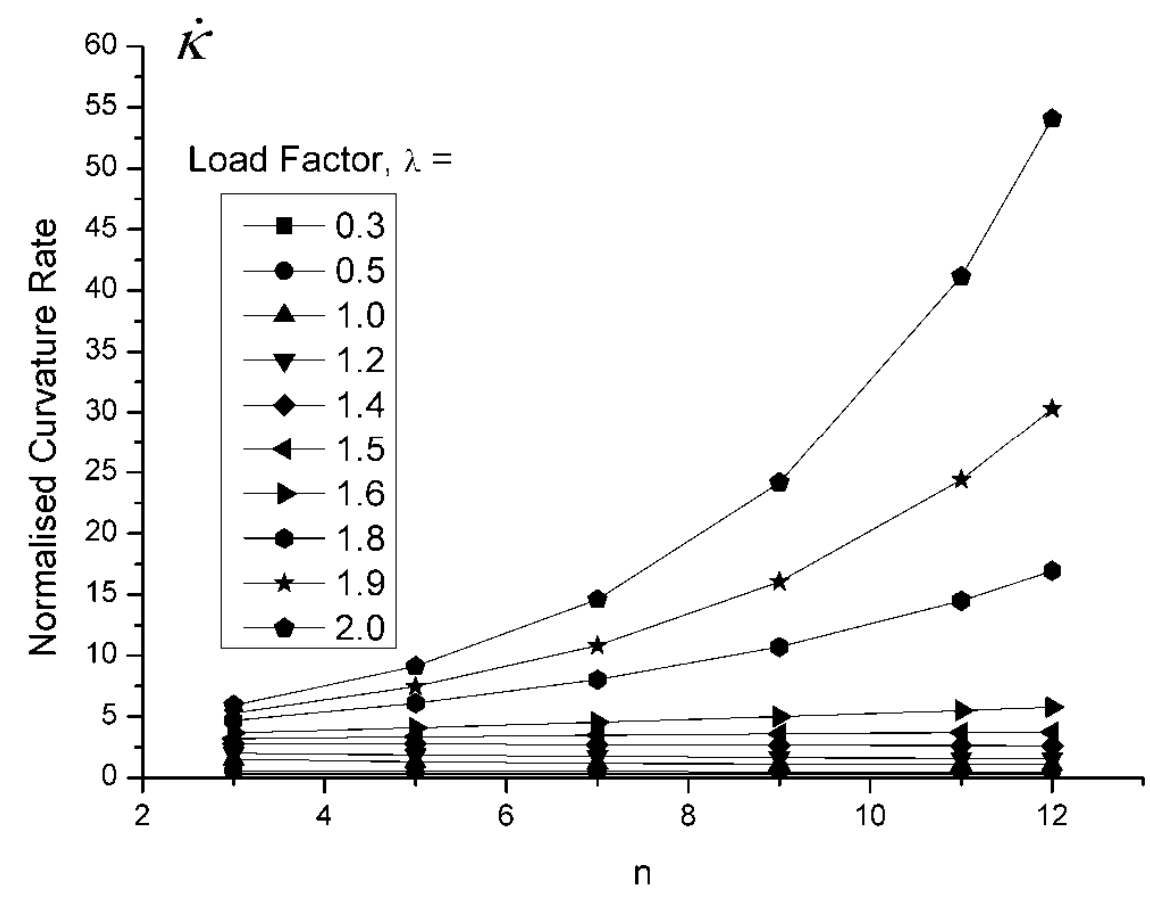

Fig. 12a Variation of maximum normalized curvature rate with $n$

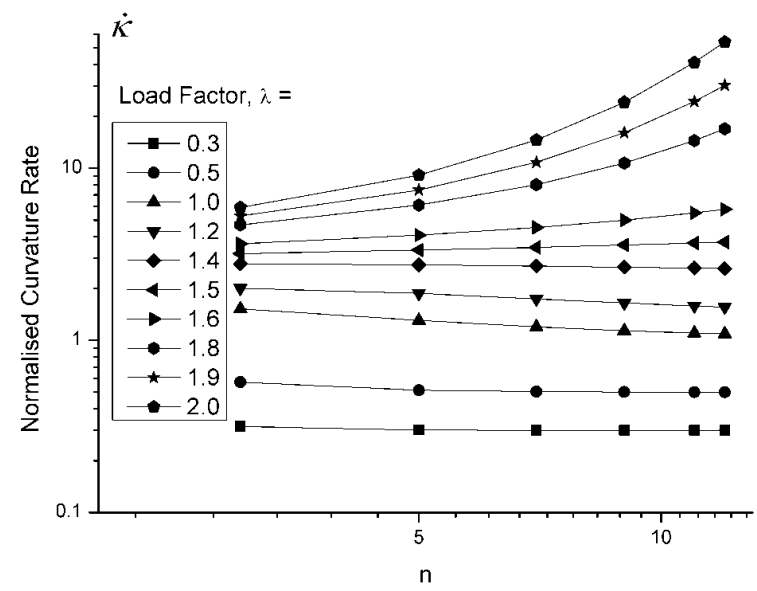

Fig. 12b Variation of maximum normalized curvature rate with $n$ : zoomed view with $\log / \log$ plot 


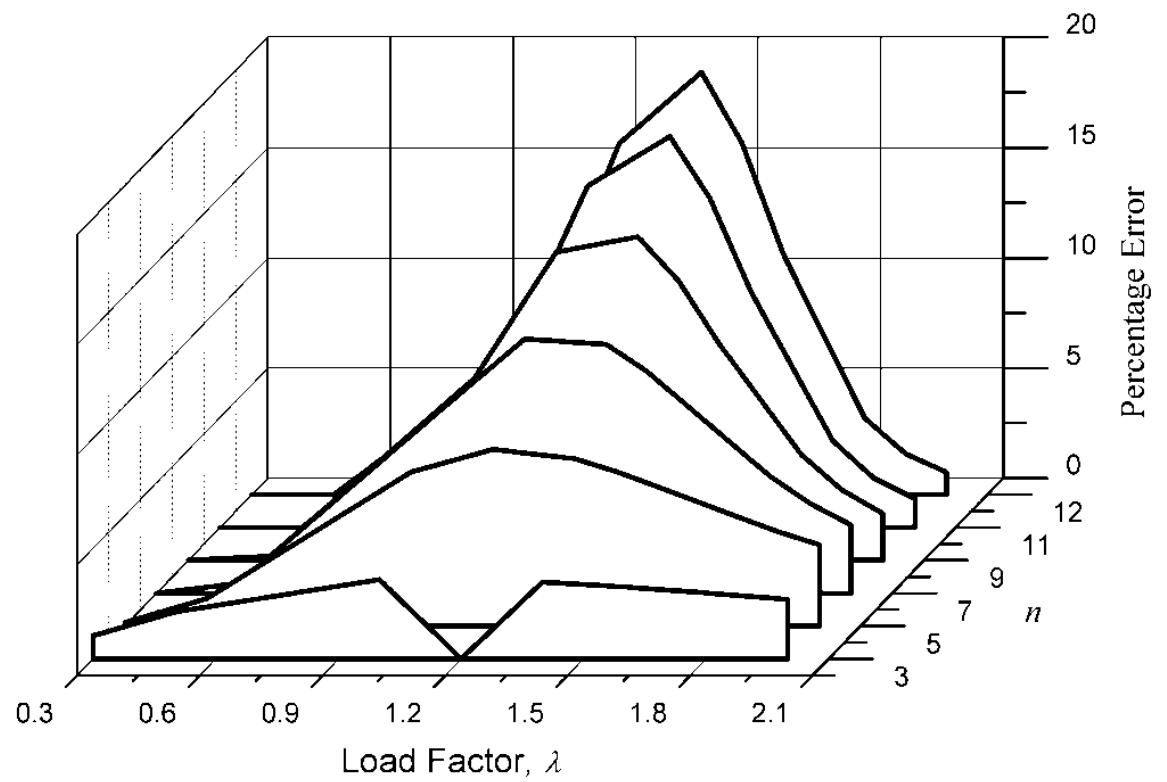

Fig. 13 Percentage error in normalized curvature rate comparing modified power law and superposition of pure linear and pure power law 


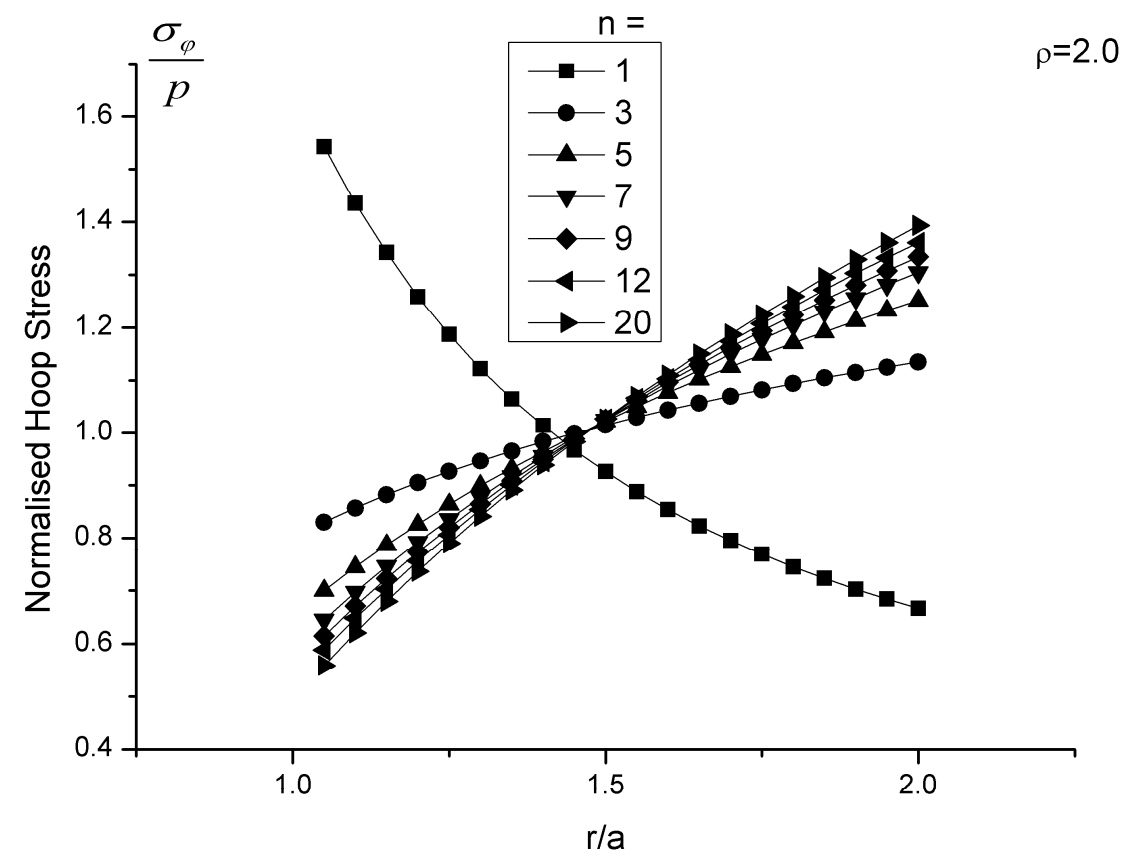

Fig. 14a Normalized hoop stress distributions in pressurized cylinder: pure linear and power law creep for $\rho=2.0$ 

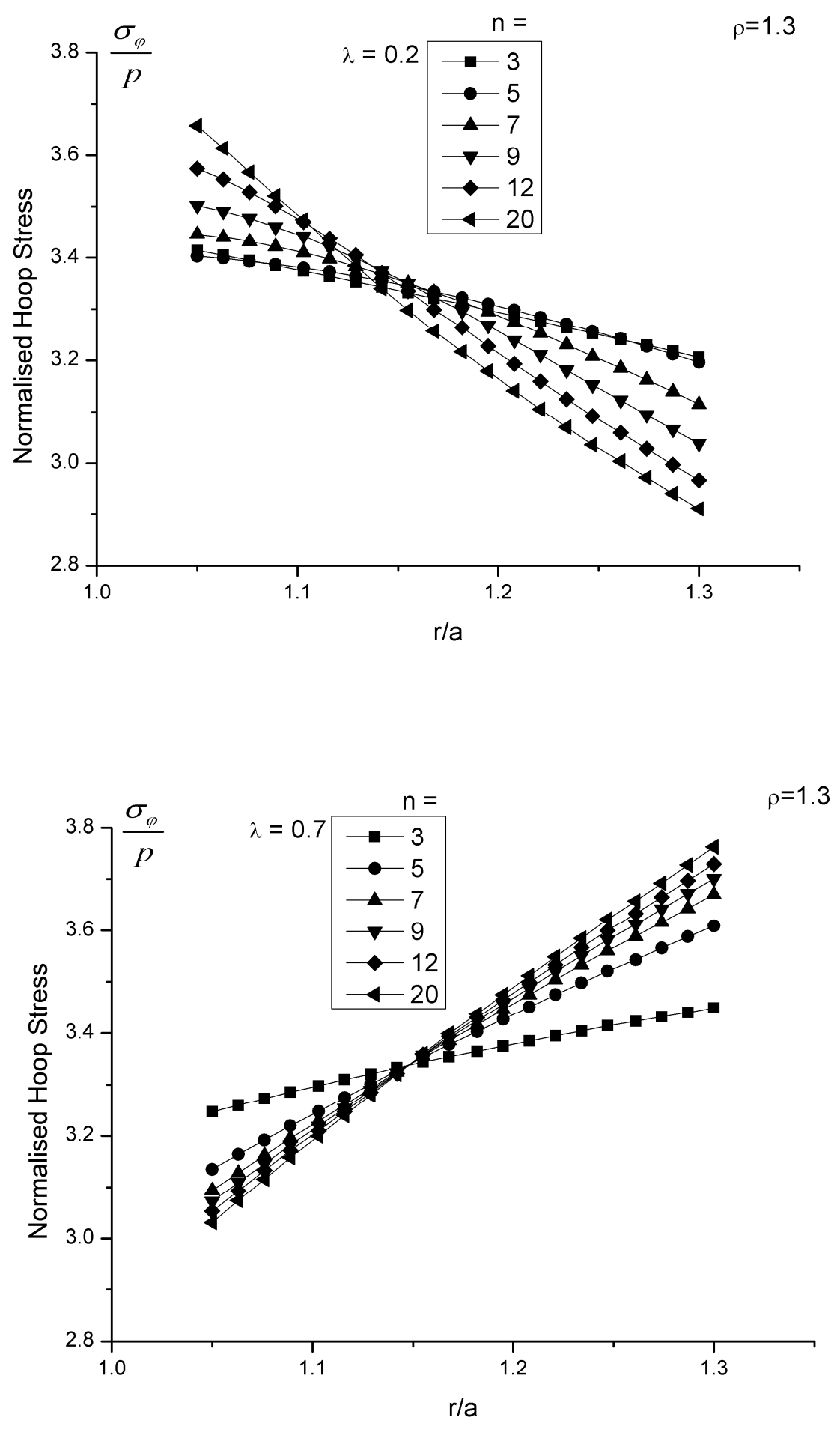

Fig. 14 b c Normalized hoop stress distributions in pressurized cylinder using modified power law, $\rho=1.3$ 

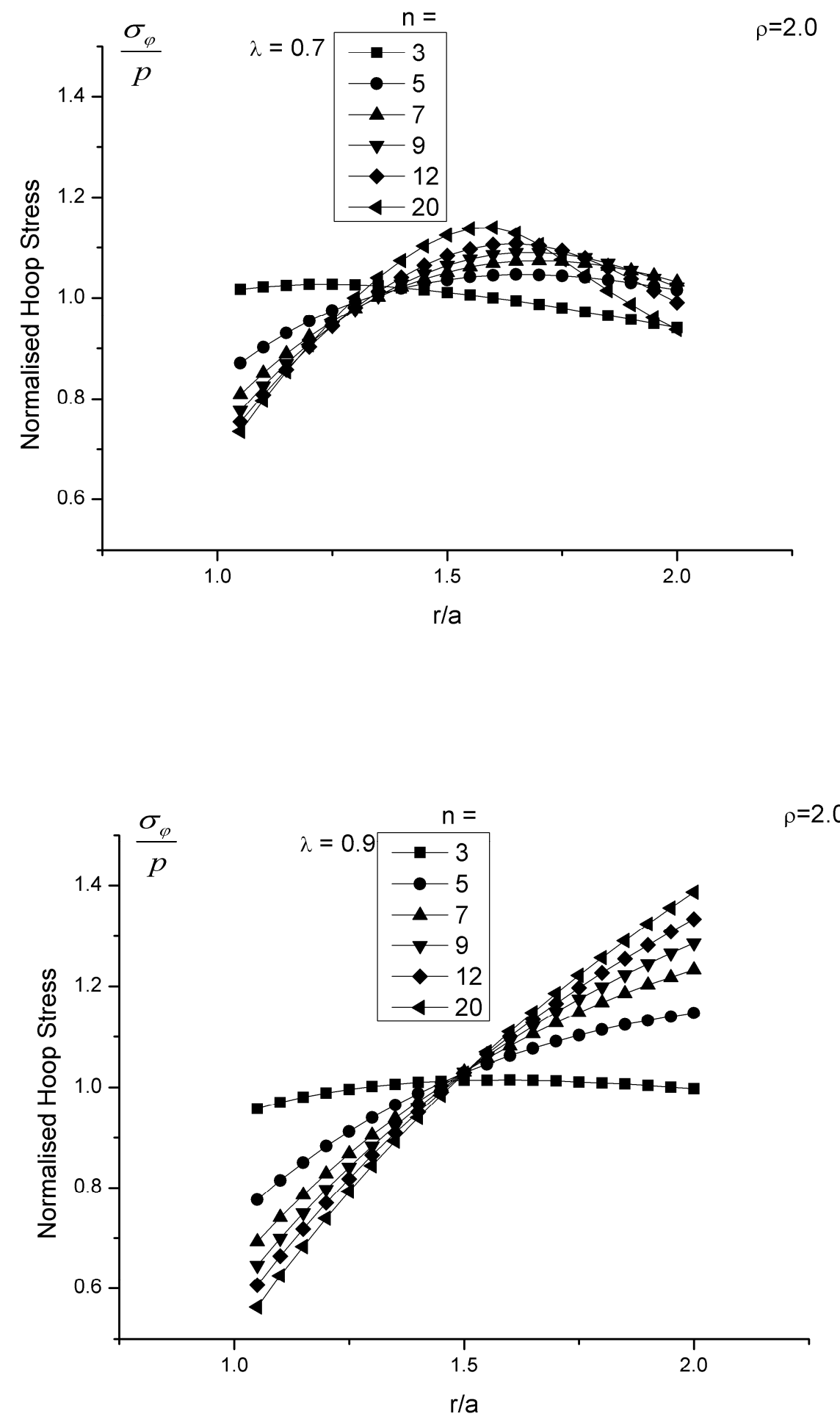

Fig. 14 d e Normalized hoop stress distributions in pressurized cylinder using modified power law, $\rho=2.0$ 

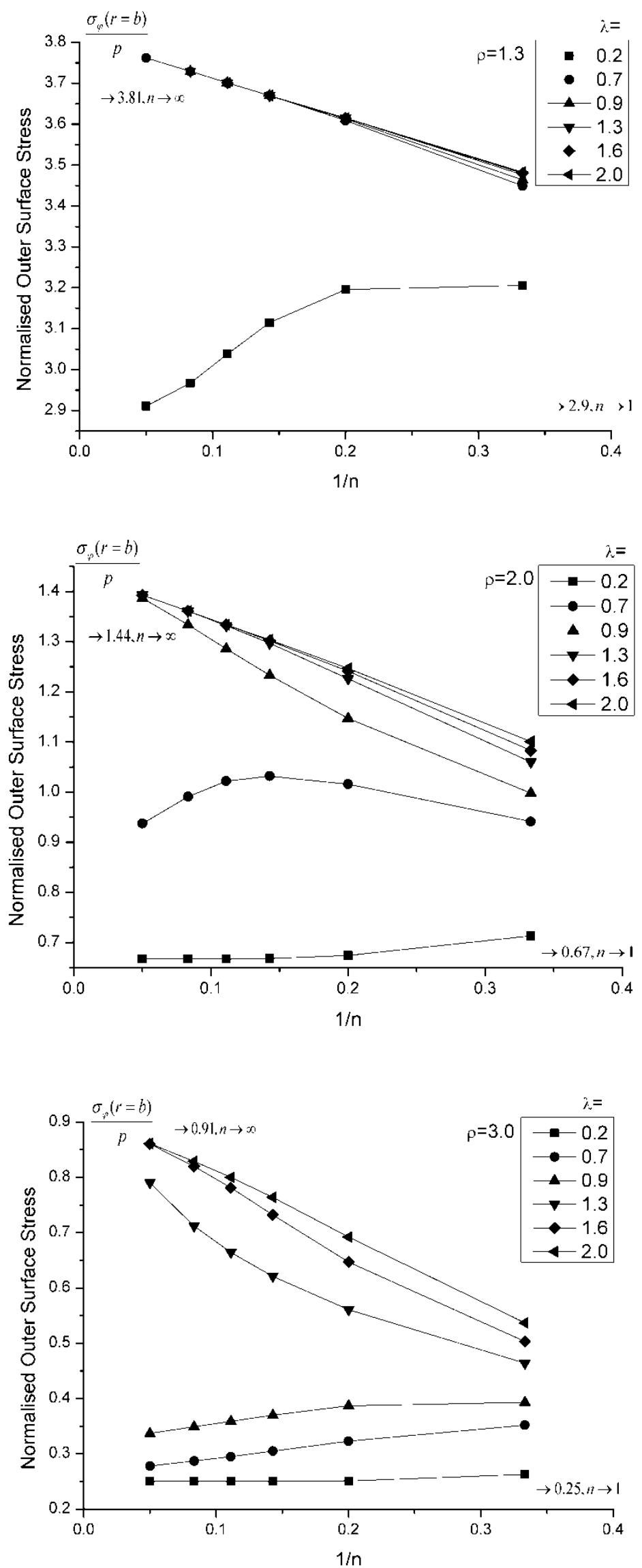

Fig. 15 Variation of outer surface hoop stress with $1 / n$ 

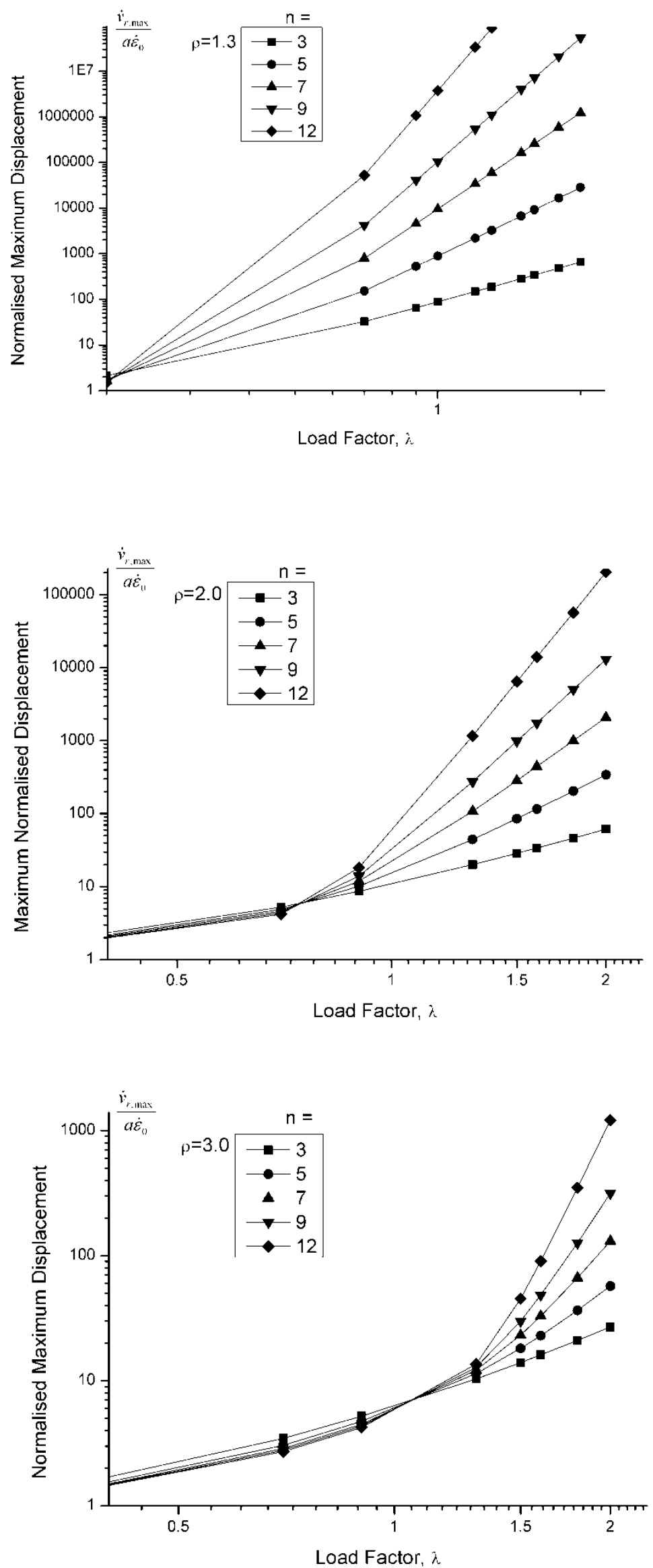

Fig. 16 Variation of maximum normalized displacement with load factor 

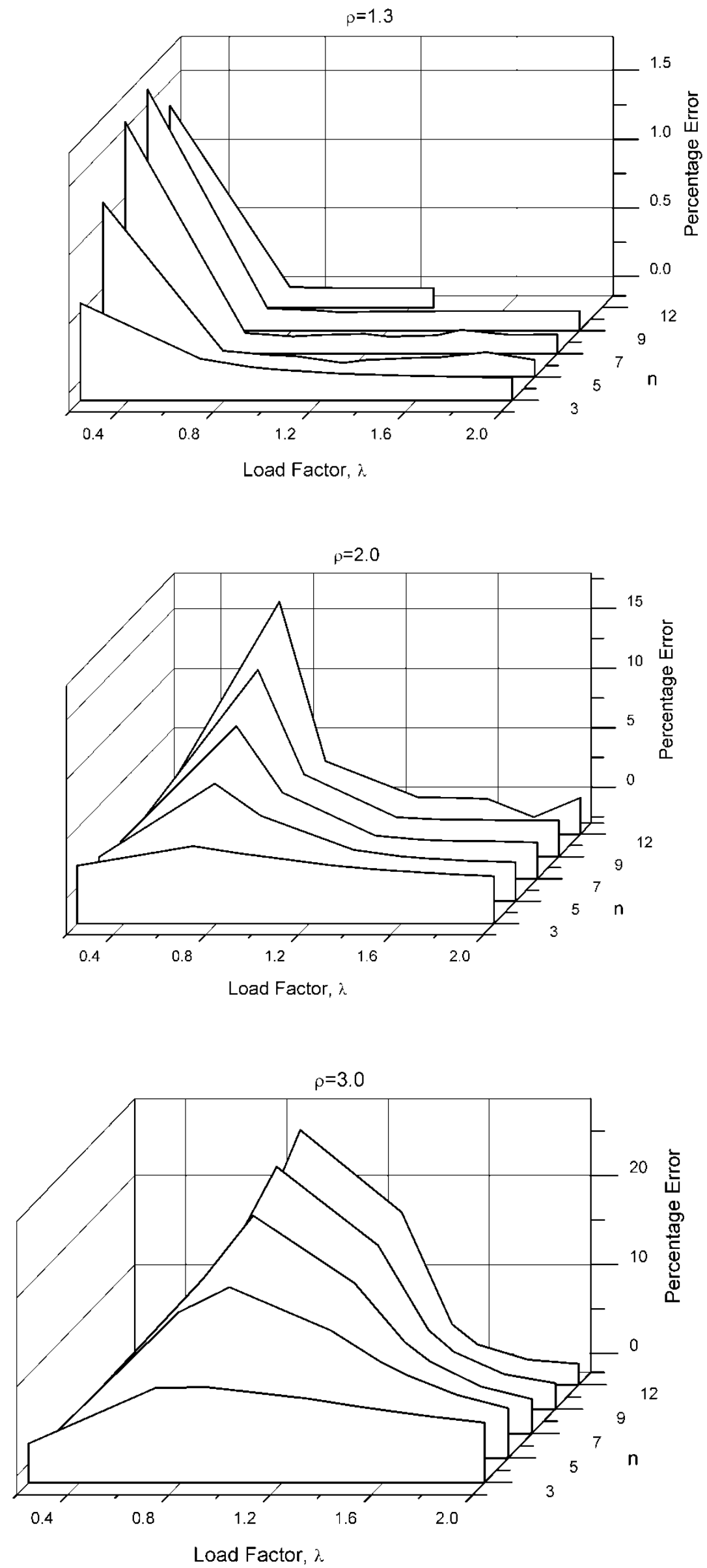

Fig. 17 Percentage error in maximum normalized displacement comparing modified power law and superposition of pure linear and pure power law 